\title{
Artificial Neural Network Based Method to Mitigate Temporary Overvoltages
}

\author{
I. Sadeghkhani ${ }^{1}{ }^{*}$, A. Ketabi ${ }^{1}$ and R. Feuillet ${ }^{2}$ \\ ${ }^{I}$ Department of Electrical Engineering, University of Kashan, Kashan, Iran \\ ${ }^{2}$ Laboratoire d'Electrotechnique de Grenoble, INPG/ENSIEG, BP46, 38402 Saint Martin d'Hères, Cedex, France
}

Received 30 December 2010;; Accepted 28 December 2011

\begin{abstract}
During early stage of primary restoration process, unexpected overvoltages may happen due to nonlinear interaction between the unloaded transformer and the transmission system. The most effective method for the limitation of the switching overvoltages is controlled switching since the magnitudes of the produced transients are strongly dependent on the closing instants of the switch. We introduce a harmonic index that it's minimum value is corresponding to the best case switching time. Also, this paper presents an Artificial Neural Network (ANN)-based approach to estimate the optimum switching instants for real time applications. In the proposed ANN, Levenberg-Marquardt second order method is used to train the multilayer perceptron. ANN training is performed based on equivalent circuit parameters of the network. Thus, trained ANN is applicable to every studied system. To verify the effectiveness of the proposed index and accuracy of the ANN-based approach, two case studies are presented and demonstrated.
\end{abstract}

Keywords: Artificial neural networks, equivalent circuit, harmonic index, temporary overvoltages, inrush currents, power system restoration, transformer energization.

\section{Introduction}

At a time when bulk power systems operate close to their design limits, the restructuring of the electric power industry has created vulnerability to potential blackouts. Prompt and effective power system restoration is essential for the minimization of downtime and costs to the utility and its customers, which mount rapidly after a system blackout $[1,2]$. A major process of power system restoration following a blackout would be energization of primary restorative transmission lines in most countries [3]. The energizing process begins by starting black-start generators such as hydro generators or gas turbines, and then charging some pre-defined transmission lines to supply cranking power for large generation plants [4,5]. Then the energization of unloaded transformers would be followed by switching action, and that is an inevitable process of bottomup restoration strategy. During transformer energization, unexpected over-voltage may happen due to nonlinear interaction between the unloaded transformer and the transmission system [1,2,6]. When a lightly loaded transformer is energized, the initial magnetizing current is generally much larger than the steady-state magnetizing current and often much larger than the rated current of the transformer [7]. Controlled switching has been recommended as a reliable method to reduce switching overvoltage during energization of capacitor banks, transformers, and transmission lines [8]. This technique is the most effective method for the limitation of the switching

\footnotetext{
*E-mail address: sadeghkhani@grad.kashanu.ac.ir

ISSN: 1791-2377 @ 2011 Kavala Institute of Technology. All rights reserved.
}

transients since the magnitudes of the created transients are strongly dependent on the closing instants of the switch [6,9].

The fundamental requirement for all controlled switching applications is the precise definition of the optimum switching instants [9]. This paper presents a novel method for controlled energization of transformers in order to minimize temporary overvoltages. We introduce a harmonic index to determine the best case switching time. Using numerical algorithm we can find the time that the harmonic index is minimum, i.e., harmonic overvoltages is minimum. Also, for real time applications, this paper presents an Artificial Neural Network (ANN)-based approach to estimate optimum switching angle during transformer energization. In the proposed ANN, LevenbergMarquardt second order method is used to train the multilayer perceptron [10,11]. The proposed ANN is expected to learn many scenarios of operation to give the optimum switching angle in a shortest computational time which is the requirement during online operation of power systems. In the proposed ANN we have considered the most important aspects, which influence the inrush currents such as voltage at transformer bus before switching, equivalent resistance, equivalent inductance, equivalent capacitance, line length, line capacitance, switching angle, and remanent flux. This information will help the operator to select the proper best-case switching condition of transformer to be energized safely with transients appearing safe within the limits.

The paper is organized as follows: in section 2 a brief description of switching overvoltages during restoration is presented. Section 3 presents modelling issues. Section 4 
describes the proposed method for best switching ccondition evaluation. In section 5 the ANN-based approach to estimate optimum switching angle during transformer energization is illustrated. Two case studies that are a portion of 39-bus New England test system are demonstrated in section 6.

\section{Switching Overvoltages during Restoration}

One of the major concerns in power system restoration is the occurrence of overvoltages as a result of switching procedures [2]. The major cause of harmonic resonance overvoltages problems is the switching of lightly loaded transformers at the end of transmission lines. After transformer energization, inrush currents with significant harmonic content up to frequencies around ten times of system frequency are produced. The harmonic current components of the same frequency as the system resonance frequencies are amplified in case of parallel resonance, thereby creating higher voltages at the transformer terminals [12]. This leads to a higher level of saturation resulting in higher harmonic components of the inrush current which again results in increased voltages. They may lead to long lasting overvoltages resulting in arrester failures and system faults and prolong system restoration [2]. This can happen particularly in lightly damped systems, common at the beginning of a restoration procedure when a path from a black-start source to a large power plant is being established and only a few loads are restored yet $[1,7,13]$.

The root cause of this phenomenon is the unfavorable combination of the source impedance, the shunt capacitance of the energized circuits, the non-linear magnetizing characteristics of the energized transformer, inadequate damping of the system and the source voltage phase angle at the moment the transformer is energized. Key factors for the harmonic overvoltages analysis can be listed as follows:

- The resonance frequency of the network;

- The system damping including the network losses, and the load connected to the network;

- The voltage level at the end of the EHV lines;

- The saturation characteristic of the transformers;

- The remanent fluxes in the core of the transformer;

- The closing time of the circuit breaker pole;

\section{Modelling Issues}

\subsection{PSB}

Simulations presented in this paper are performed using the PSB. The simulation tool has been developed using state variable approach and runs in the MATLAB/Simulink environment. This program has been compared with other popular simulation packages (EMTP and Pspice) in [14]. The user friendly graphical interfaces of PSB enable faster development for power system transient analysis.

\subsection{Generator Model}

In [15] generators have been modeled by generalized Park's model that both electrical and mechanical part are thoroughly modeled, but it has been shown that a simple static generator model containing an ideal voltage source behind the sub-transient inductance in series with the armature winding resistance can be as accurate as the Park model. Thus in this work, generators are represented by the static generator model. Phases of voltage sources are determined by the load flow results.

\subsection{Transmission-Line Model}

Transmission lines are described by the distributed line model. This model is accurate enough for frequency dependent parameters, because the positive sequence resistance and inductance are fairly constant up to approximately $1 \mathrm{KHz}$ [16] which cover the frequency range of harmonic overvoltages phenomena.

\subsection{Transformer Model}

The transformer model takes into account the winding resistances $\left(R_{1}, R_{2}\right)$, the leakage inductances $\left(L_{1}, L_{2}\right)$ as well as the magnetizing characteristics of the core, which is modeled by a resistance, $\mathrm{R}_{\mathrm{m}}$, simulating the core active losses and a saturable inductance, $\mathrm{L}_{\mathrm{sat}}$. The saturation characteristic is specified as a piece-wise linear characteristic [7]. For the target transformer, hysteresis is added, in order to take into account the remanent fluxes in the iron core. The remanent fluxes in the transformer core can be obtained via the integration of the voltages measured on the transformer windings during its disconnection. The correct estimation of the residual flux is extremely important for the success of the controlled switching strategy.

\subsection{Load and Shunt Devices Model}

All of the loads and shunt devices, such as capacitors and reactors, are modeled as constant impedances.

\section{Proposed Method for Best Switching Condition Evaluation}

The main part of a controlled switching arrangement is a controller, which is the "brain" of the system. It receives the signals from the measuring devices, determines the appropriate reference phase angles and sends the switching commands to each pole of the switching device so that closing operation occurs at the optimum instant.

\subsection{Calculation of Transformer inrush Current}

In order to analyze the transformer inrush current, let us consider a single-phase transformer having a magnetizing characteristic as given in Fig. 1. If the voltage applied to the transformer is $v=V \cos \omega t$ at time $\mathrm{t}_{0}$ for which $\omega \mathrm{t}_{0}=\theta$, the established flux linkage will be a function of the applied voltage and the flux linkage offset $\psi_{0}$ as given by the following expression [5]: 


$$
\psi=\frac{V}{\omega} \sin \omega t+\psi_{0}
$$

The flux linkage offset is made up of the remanent flux linkage $\psi_{r}$ and the component $-V / \omega \sin \theta$ due to the linkage mismatch condition at energization,

$$
\psi_{0}=\psi_{r}-\frac{V}{\omega} \sin \theta
$$

Under normal system conditions the transformer core would be driven temporarily into saturation asymmetrically when the flux linkage $\psi$ exceeds the saturation level $\psi_{s}$. The expression for the inrush current is:

$$
i=\frac{1}{L_{s a t}}\left(\psi-\psi_{s}\right)
$$

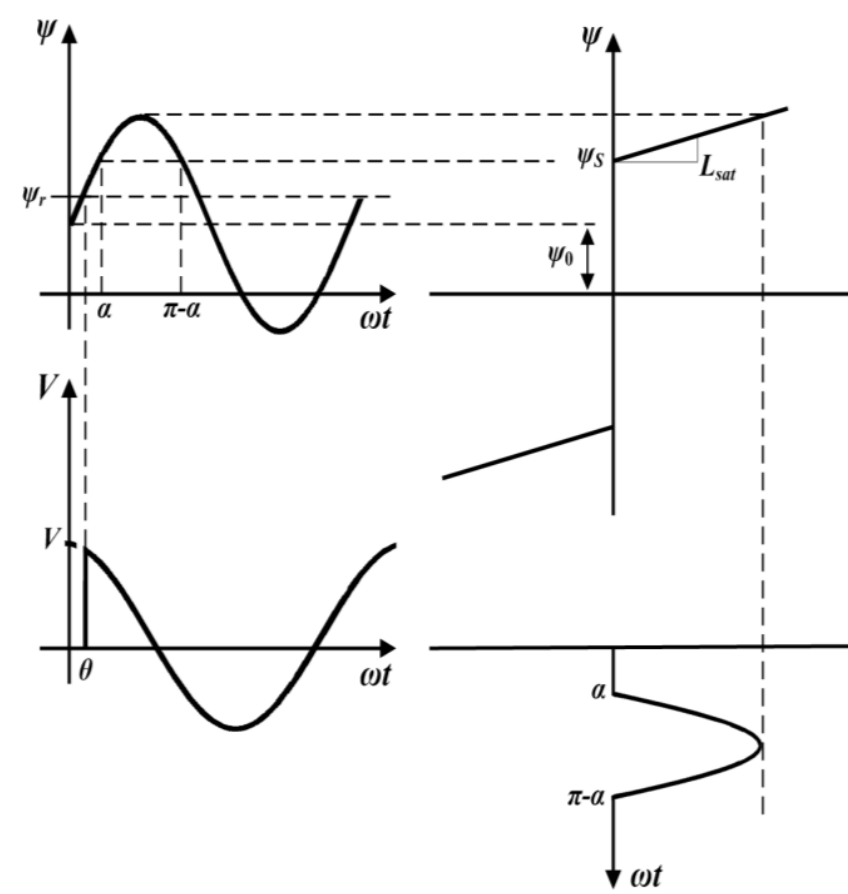

Fig. 1. Simplified transformer magnetization characteristic.

Combining (1) to (3), the following expression is obtained for the inrush current (in p.u.):

$$
\begin{aligned}
\frac{i}{I_{s}} & =\frac{V}{V_{s}} \sin \omega t+s-1 & & \text { for } \quad \alpha \leq \omega t \leq \pi-\alpha \\
& =0 & & \text { elsewhere }
\end{aligned}
$$

where:

$$
\alpha=\sin ^{-1}\left[\left(1-\frac{\psi_{0}}{\psi_{s}}\right) \frac{V_{s}}{V}\right]
$$

$$
I_{s}=\frac{V_{s}}{\omega L_{s a t}}
$$

$$
s=\frac{\psi_{0}}{\psi_{s}}
$$

Fourier analysis of the inrush current yields the following expressions for it's harmonic components:

$$
\frac{I_{0}}{I_{s}}=\frac{1}{2 \pi}[2 \cos \alpha-(2 \alpha-\pi) \sin \alpha] \frac{V}{V_{s}}
$$

$$
\frac{I_{1}}{I_{s}}=\frac{1}{2 \pi}[\pi-2 \alpha-\sin 2 \alpha] \frac{V}{V_{s}}
$$

$$
\frac{I_{k}}{I_{s}}=\frac{1}{k \pi}\left[\frac{\sin [(k-1)(\alpha+\pi / 2]}{k-1}-\frac{\sin [(k+1)(\alpha+\pi / 2]}{k+1}\right] \frac{V}{V_{s}}
$$$$
k=2,3,4, \ldots
$$

where $\psi_{0}$ is the flux linkage offset, $L_{s}$ is the transformer saturation inductance, $\psi_{r}$ is the initial residual flux, and $\psi_{s}$ and $V_{s}$ are transformer saturation flux linkage and

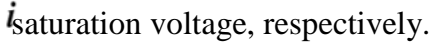

\subsection{Best Switching Condition Determination}

Normally for harmonic overvoltages analysis, the best case of the switching condition must be considered which it is a function of switching time, transformer characteristics and its initial flux condition, and impedance characteristics of the $\rightarrow$ witching bus. Using the best switching condition, the harmonic overvoltages peak and duration can be reduced significantly.

In order to determine best-case switching time, the following index is defined as

$W=\sum_{h=2}^{10} Z_{j j}(h) \cdot I_{j}\left(h, t_{0}, \phi_{r}\right)$

This index can be a definition for the best-case switching condition. Using a numerical algorithm, one can find the switching time for which $\mathrm{W}$ is minimal (i.e., harmonic overvoltages is minimal).

The sample system considered for explanation of the proposed methodology is a $400 \mathrm{kV}$ EHV network shown in Fig. 2. The normal peak value of any phase voltage is $400 \sqrt{2} / \sqrt{3} \mathrm{kV}$ and this value is taken as base for voltage p.u. In the system studies $400 \mathrm{kV}$ line-to-line base voltage and $100 \mathrm{MVA}$ as a base power is considered. 


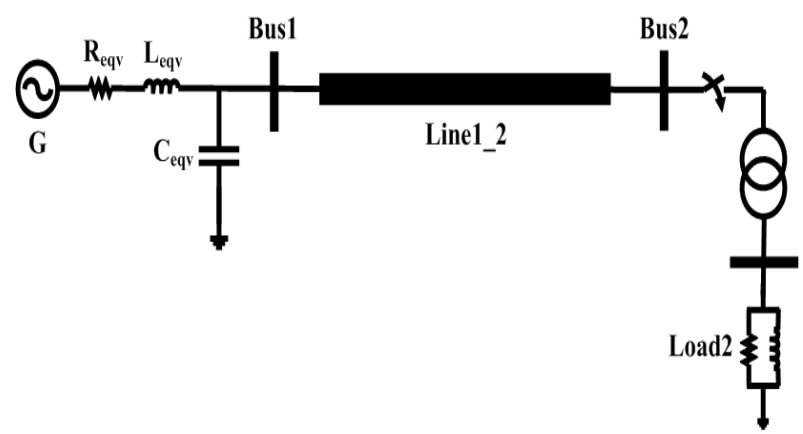

Fig. 2. Sample system for transformer energization study. G: generator, $\mathrm{R}_{\text {eqv }}$ : equivalent resistance, $\mathrm{L}_{\text {eqv }}$ : equivalent inductance, and $\mathrm{C}_{\text {eqv }}$ : equivalent capacitance.

In this paper equivalent circuit parameters are used as ANN inputs together other parameters to achieve good generalization capability for trained ANN. In fact, in this approach ANN is trained just once for sample system of Fig. 2. Since ANN training is based on equivalent circuit parameters, developed ANN is applicable to every studied system. This issue is better understood in section 6 that trained ANN is tested for a 39-bus New England test system.

Fig. 3 shows the result of the frequency analysis at bus 2 . The magnitude of the Thevenin impedance, seen from bus 2 , $\mathrm{Z}_{\text {bus2 }}$ shows a parallel resonance peak at $230 \mathrm{~Hz}$. Fig. 4 shows changes of harmonic currents and $\mathrm{W}$ index with respect to the switching angle, where $\mathrm{k}$ is harmonic number. Fig. 5 shows the harmonic overvoltages after the transformer energization for the best-case condition (i.e., 56 ${ }^{\circ}$ ). For temporary overvoltages, the overvoltage duration has to be taken into account in addition to the amplitude [3]. Table 1 summarizes the results of overvoltages simulation for five different switching conditions that verify the effectiveness of $\mathrm{W}$ index.

In the next section we present an Artificial Neural Network (ANN)-based approach to estimate optimum switching angle for real time applications.

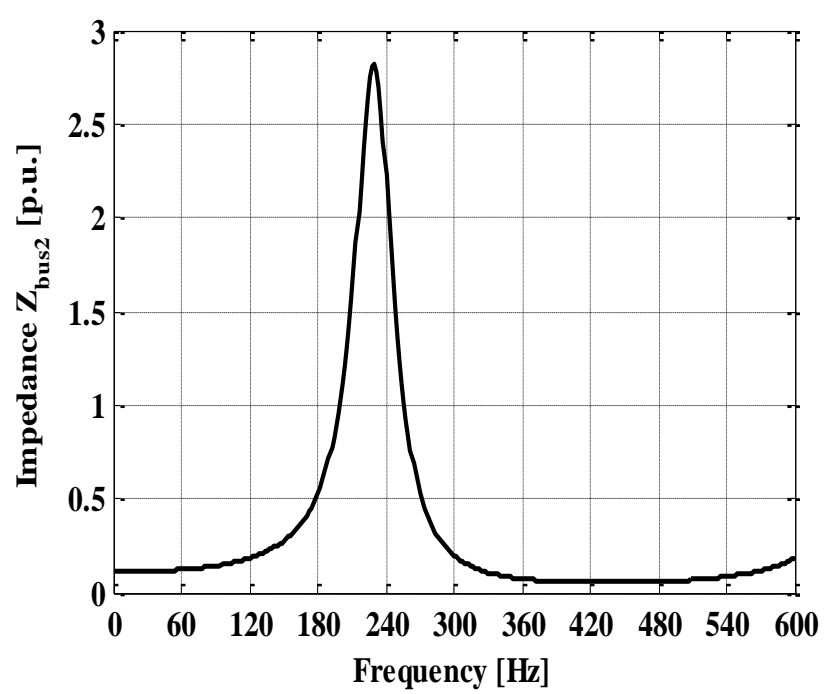

Fig. 3. Impedance at bus 2 .

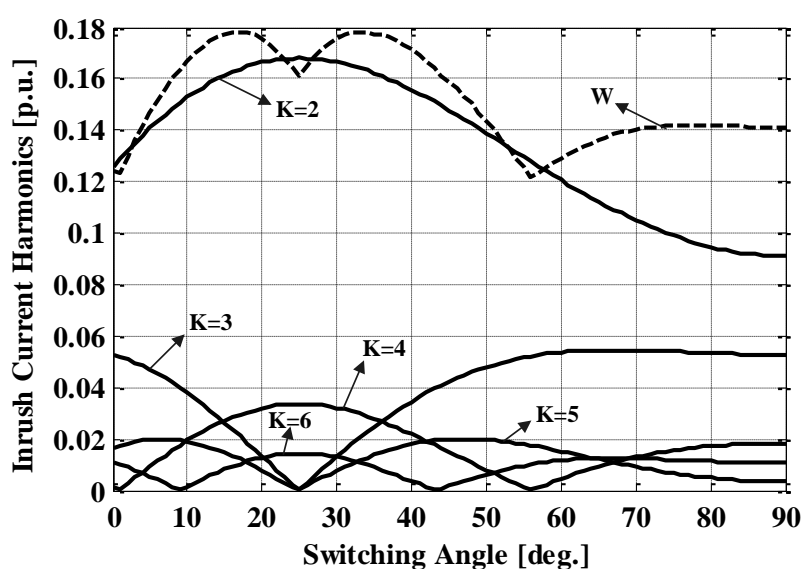

Fig. 4. Changes of harmonic currents and $\mathrm{W}$ index with respect to the switching angle.

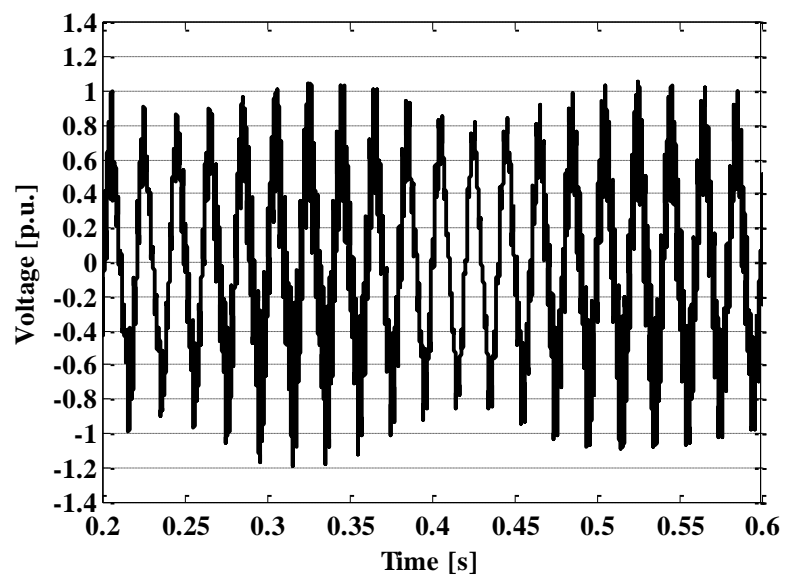

Fig. 5. Voltage at bus 2 after switching of transformer for best switching condition.

Table 1. Effect of switching time on the minimum of overvoltages and duration of $\mathrm{V}_{\text {peak }}>1.3$ p.u.

\begin{tabular}{lll}
\hline $\begin{array}{l}\text { Switching Angle } \\
\text { [deg.] }\end{array}$ & $\mathbf{V}_{\text {peak }}$ [p.u.] & $\begin{array}{l}\text { Duration of }\left(\mathbf{V}_{\text {peak }}>\mathbf{1 . 3}\right. \\
\text { p.u. })[\mathbf{s}]\end{array}$ \\
\hline 56 & 1.1857 & 0 \\
45 & 1.5104 & 0.3752 \\
33 & 1.6527 & 0.4253 \\
70 & 1.3892 & 0.1442 \\
10 & 1.5861 & 0.3248 \\
\hline
\end{tabular}

\section{The Artificial Neural Network}

The proposal in this work considers the adoption of feed forward Multilayer Perceptron (MLP) architecture. A MLP trained with the back-propagation algorithm may be viewed as a practical vehicle for performing a nonlinear inputoutput mapping of a general nature [10,17]. Function approximation by feed forward MLP network is proven to be very efficient, considering various learning strategies like simple back propagation or the robust LevenbergMarquardt. Its ability to perform well is affected by the 
chosen training data as well as training scheme.

As mentioned in previous section, following parameters are effective in determination of optimum switching angle during transformer energization:

- Voltage at transformer bus before switching

- Equivalent resistance of the network

- Equivalent inductance of the network

- Equivalent capacitance of the network

- Line length

- Line capacitance

- Closing time of the circuit breaker poles

- Remanent flux

The schematic diagram of the proposed MLP neural networks architecture is shown in Fig. 6. The composition of the input variables for the proposed neural networks has been carefully selected.

Supervised training of ANN is a usual training paradigm for MLP architecture. Fig. 7 shows the supervised learning of ANN for which input is given to proposed method to get the optimum switching angle values and the same data is used to train the ANN. Error is calculated by the difference of proposed method output and ANN output. This error is used to adjust the weight of connection. Output values of the trained neural networks must be capable of computing the optimum switching angle with very good precision. Gradient-based training algorithms, like back propagation, are most commonly used for training procedures. They are not efficient due to the fact that the gradient vanishes at the solution. Hessian-based algorithms allow the network to learn more subtle features of a complicated mapping. The training process converges quickly as the solution is approached, because the Hessian does not vanish at the solution. To benefit from the advantages of Hessian based training, we focused on the Levenberg-Marquardt (LM) algorithm reported in [11].

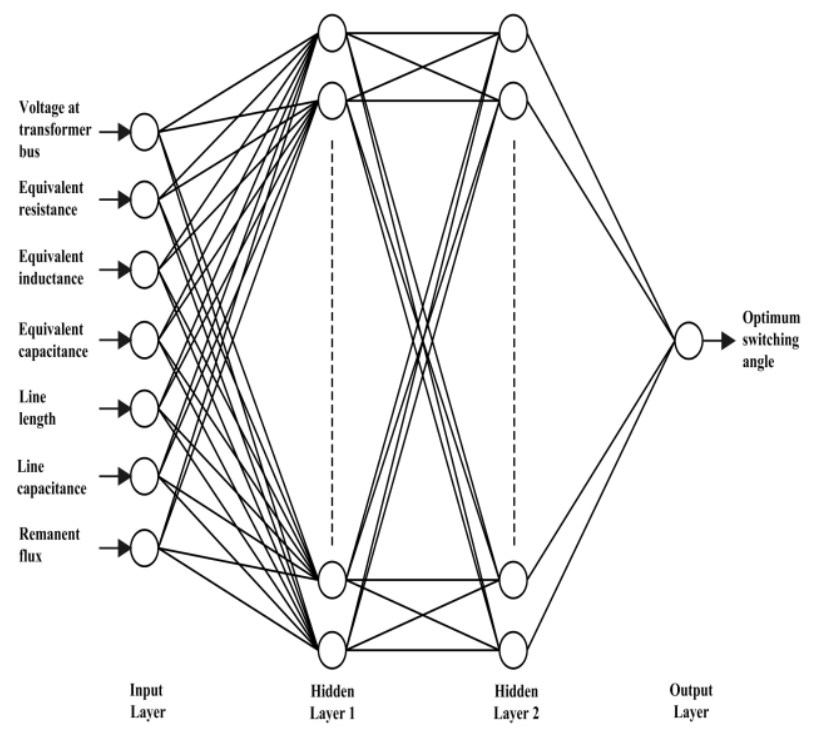

Fig. 6. Proposed MLP-based ANN architecture.

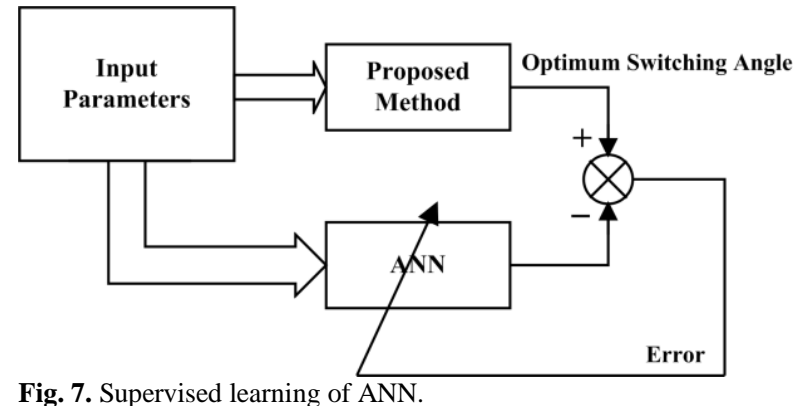

\subsection{Levenberg-Marquardt (LM) Algorithm}

Suppose that we have a function $\xi(\mathbf{x})$ which we want to minimize with respect to the parameter vector $\mathbf{x}$, where

$$
\xi(\mathbf{x})=\sum_{i=1}^{N} e_{i}^{2}(\mathbf{x})
$$

Then the Marquardt-Levenberg modification to the Gauss-Newton method is

$$
\Delta \mathbf{x}=\left[\mathbf{J}^{\mathrm{T}}(\mathbf{x}) \mathbf{J}(\mathbf{x})+\mu \mathbf{I}\right]^{-1} \mathbf{J}^{\mathrm{T}}(\mathbf{x}) \mathbf{e}(\mathbf{x})
$$

The parameter $\mu$ is multiplied by some factor $\beta$ whenever a step would result in an increased $\xi(\mathbf{x})$. When a step reduces $\xi(\mathbf{x}), \mu$ is divided by $\beta$. Notice that when $\mu$ is large the algorithm becomes steepest descent; while for small $\mu$ the algorithm becomes Gauss-Newton. The LM algorithm is very efficient when training networks have up to few hundred weights. Although the computational requirements are much higher for the each iteration of the LM algorithm, this is more than made up for by the increased efficiency. This is especially true when high precision is required.

\subsection{Steps of Optimum Switching Angle Estimation}

The steps for optimum switching angle evaluation and estimation are listed below:

1) Determine the characteristics of transformer that must be energized.

2) Calculate the $Z_{\mathrm{ii}}(\mathrm{h})$ at the transformer bus for $\mathrm{h}=$ $2 \mathrm{f}_{0}, \ldots, 10 \mathrm{f}_{0}$.

3) Calculate the best switching condition.

4) Repeat the above steps with various system parameters to learn artificial neural network.

5) Test artificial neural network with different system parameters.

\subsection{Training Artificial Neural Network}

All experiments have been repeated for different system parameters. After learning, all parameters of the trained networks have been frozen and then used in the retrieval mode for testing the capabilities of the system on the data not used in learning. The testing data samples have been generated through the proposed method by placing the parameter values not used in learning, by applying different parameters. A large number of testing data have been used to check the proposed solution in the most objective way at 
practically all possible parameters variation. Percentage error is calculated as:

$\operatorname{error}(\%)=\frac{\mid \text { ANN }- \text { Method } \mid}{\text { Method }} \times 100$

Neural network is trained with the goal of mean square error (MSE) 1e-2. Fig. 8 shows the training of neural network. Results for a sample test data are presented in Table 2.

In the next section, the proposed model tested with portion of 39-bus New England test system.

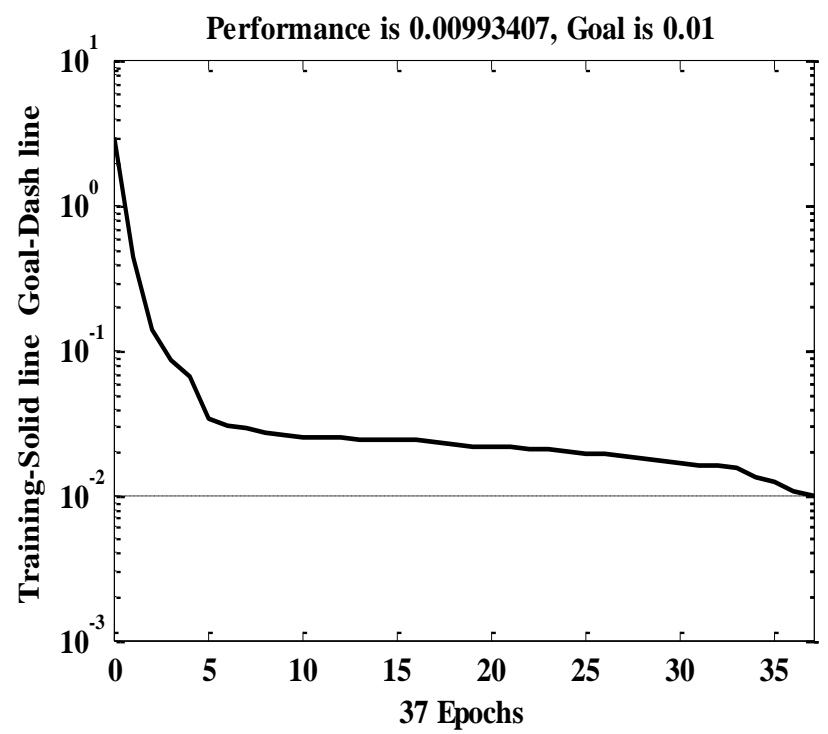

Fig. 8. Squared error against epoch curve.

Table 2. Some sample testing data and output

\begin{tabular}{|c|c|c|c|c|c|c|c|c|c|}
\hline $\begin{array}{c}\mathbf{V} \\
\text { [p.u.] }\end{array}$ & $\begin{array}{c}\mathbf{R}_{\mathrm{eqv}} \\
\text { [p.u.].] }\end{array}$ & $\begin{array}{c}\mathbf{L}_{\text {eqv }} \\
\text { [p.u.] }\end{array}$ & $\begin{array}{c}\mathbf{C}_{\text {eqv }} \\
\text { [p.u.] }\end{array}$ & $\begin{array}{l}\text { L.L. } \\
\text { [km] }\end{array}$ & $\begin{array}{c}\mathbf{C}_{\text {Line }} \\
{[\mathbf{F} / \mathbf{k m}]}\end{array}$ & $\begin{array}{c}\boldsymbol{\Phi}_{\mathbf{r}} \\
{[\mathbf{p} . \text {.u. }]}\end{array}$ & $\begin{array}{l}\text { B.S.A.HI } \\
\text { [deg.] }\end{array}$ & $\begin{array}{l}\text { B.S.A.A.AvN } \\
\text { [deg.] }\end{array}$ & $\begin{array}{c}\text { Error } \\
{[\%]}\end{array}$ \\
\hline 0.9077 & 0.003 & 0.0375 & 0.3694 & 100 & $\begin{array}{c}1.199 \mathrm{e}- \\
8\end{array}$ & 0.1 & 43.5 & 44.3 & 1.8526 \\
\hline 0.9254 & 0.0035 & 0.035 & 0.3694 & 125 & $\begin{array}{c}1.199 \mathrm{e}- \\
8\end{array}$ & 0.2 & 28.1 & 28.4 & 1.1637 \\
\hline 0.9731 & 0.004 & 0.0325 & 0.9781 & 150 & $\begin{array}{c}1.224 \mathrm{e}- \\
8\end{array}$ & 0.3 & 58.6 & 56.4 & 3.8201 \\
\hline 0.9973 & 0.0045 & 0.03 & 0.9781 & 180 & $\begin{array}{c}1.224 \mathrm{e}- \\
8\end{array}$ & 0.3 & 41.9 & 42.9 & 2.28 \\
\hline 1.0426 & 0.005 & 0.0275 & 1.2825 & 200 & $\begin{array}{c}1.237 \mathrm{e}- \\
8\end{array}$ & 0.4 & 88.4 & 86.7 & 1.97 \\
\hline 1.0621 & 0.0055 & 0.0275 & 1.2825 & 220 & $\begin{array}{c}1.237 \mathrm{e}- \\
8\end{array}$ & 0.5 & 75.6 & 72.3 & 4.3947 \\
\hline 1.1222 & 0.006 & 0.025 & 1.5869 & 250 & $\begin{array}{c}1.249 \mathrm{e}- \\
8\end{array}$ & 0.6 & 51.3 & 50.6 & 1.3528 \\
\hline 1.1224 & 0.003 & 0.0225 & 1.5869 & 250 & $\begin{array}{c}1.249 \mathrm{e}- \\
8\end{array}$ & 0.7 & 25.7 & 26.1 & 1.6914 \\
\hline 1.1668 & 0.005 & 0.02 & 2.1956 & 265 & $\begin{array}{c}1.274 \mathrm{e}- \\
8\end{array}$ & 0.7 & 90 & 89.1 & 0.9525 \\
\hline 1.1824 & 0.0035 & 0.02 & 2.1956 & 280 & $\begin{array}{c}1.274 \mathrm{e}- \\
8\end{array}$ & 0.8 & 48.5 & 49.6 & 2.3691 \\
\hline
\end{tabular}

\section{Case Study}

In this section, the proposed algorithm is demonstrated for two case studies that are a portion of 39-bus New England test system, which its parameters are listed in [18]. The simulations are undertaken on a single phase representation.

\subsection{Case 1}

Fig. 9 shows a one-line diagram of a portion of 39-bus New England test system which is in restorative state. The generator at bus 35 is a black-start unit. The load 19 shows cranking power of the later generator that must be restored by the transformer of bus 19 . When the transformer is energized, harmonic overvoltages can be produced because the transformer is lightly loaded.

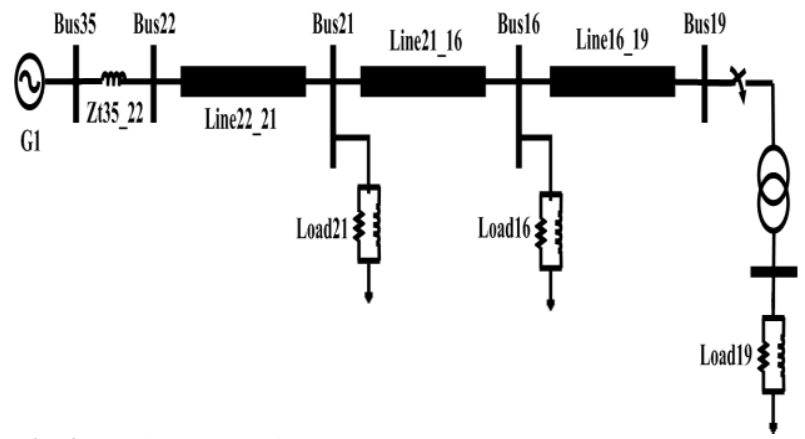

Fig. 9. Studied system for case 1.

As mentioned in section 4, first, equivalent circuit of this system, seen behind bus 16 , is determined and values of equivalent resistance, equivalent inductance, and equivalent capacitance are calculated, in other words, this system is converted to equivalent system of Fig. 2. In this case, values of equivalent resistance, equivalent inductance and equivalent capacitance are 0.00291 p.u., 0.02427, and 2.474 p.u., respectively. For testing trained ANN, values of voltage at transformer bus (bus 19), line length, and remanent flux are varied and in each step, optimum switching angle values are calculated from trained ANN and proposed method. Table 3 contains the some sample result of test data of case 1.

Table 3. Case 1 some sample testing data and output

\begin{tabular}{|c|c|c|c|c|c|}
\hline $\mathrm{V}$ [p.u.] & $\begin{array}{l}\text { L.L. } \\
\text { [km] }\end{array}$ & $\Phi_{\mathrm{r}}[\mathrm{p} . \mathrm{u}]$. & $\begin{array}{l}\text { B.S.A.HII } \\
\text { [deg.] }\end{array}$ & $\begin{array}{c}\begin{array}{c}\text { B.S.A.A.ANN } \\
\text { [deg.] }\end{array} \\
\end{array}$ & Error [\%] \\
\hline 0.9243 & 100 & 0.2 & 80.6 & 78.9 & 2.1675 \\
\hline 0.9541 & 150 & 0.3 & 37.5 & 38.2 & 1.8044 \\
\hline 1.0195 & 200 & 0.4 & 18.3 & 18.7 & 2.2783 \\
\hline 1.0481 & 230 & 0.4 & 44.8 & 45.4 & 1.2459 \\
\hline 1.0977 & 250 & 0.5 & 62.1 & 61.1 & 1.6623 \\
\hline 1.0977 & 250 & 0.6 & 89.7 & 85.6 & 4.5391 \\
\hline 1.1505 & 270 & 0.7 & 67.7 & 66.4 & 1.8542 \\
\hline 1.1776 & 290 & 0.8 & 32.6 & 32.2 & 1.2658 \\
\hline
\end{tabular}

$\mathrm{V}=$ voltage at transformer bus before switching, L.L. = line length, $\Phi_{\mathrm{r}}=$ remanent flux, B.S. $A_{\mathrm{HI}}=$ the best switching angle obtained by the harmonic index, B.S. $A_{\mathrm{ANN}}=$ the best switching angle obtained by the $\mathrm{ANN}$, and Error $=$ switching angle error.

\subsection{Case 2}

As another example, the system in Fig. 10 is examined. In the next step of the restoration, unit at bus 6 must be restarted. In order to provide cranking power for this unit, 
the transformer at bus 6 should be energized. In this condition, harmonic overvoltages can be produced because the load of the transformer is small.

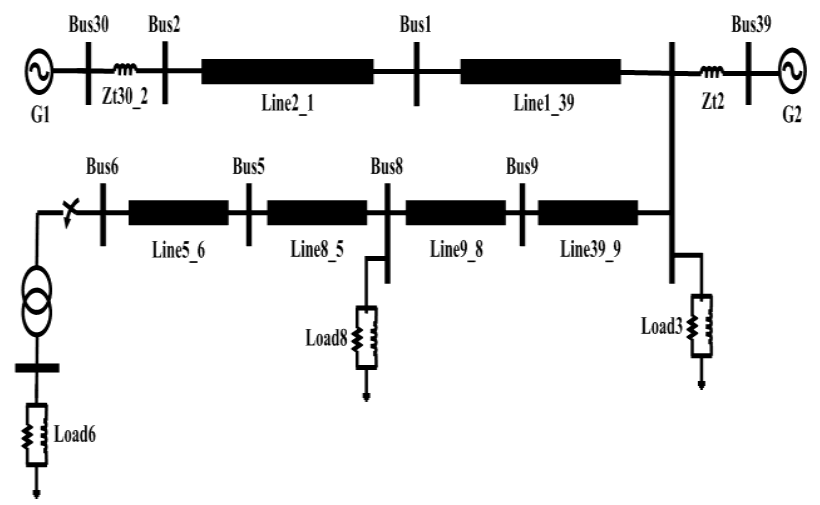

Fig. 10. Studied system for case 2 .

After converting this system to equivalent circuit of Fig. 2 , i.e., after calculating equivalent circuit seen from bus 5 , various cases of transformer energization are taken into account and corresponding optimum switching angles are computed from proposed method and trained ANN. In this case, values of equivalent resistance, equivalent inductance and equivalent capacitance are 0.00577 p.u., 0.02069 , and 0.99 p.u., respectively. Summery of few result are presented in Table 4. It can be seen from the results that the ANN is able to learn the pattern and give results to acceptable accuracy.
Table 4. Case 2 some sample testing data and output

\begin{tabular}{cccccc}
\hline V [p.u.] & $\begin{array}{c}\text { L.L. } \\
{[\mathbf{k m}]}\end{array}$ & $\boldsymbol{\Phi}_{\mathbf{r}}$ [p.u.] & $\begin{array}{c}\text { B.S.A.HI } \\
\text { [deg.] }\end{array}$ & $\begin{array}{c}\text { B.S.A.ANN } \\
\text { [deg.] }\end{array}$ & Error [\%] \\
\hline 0.9335 & 125 & 0.8 & 75.4 & 73.4 & 2.6384 \\
0.9512 & 155 & 0.7 & 42.9 & 41.4 & 3.5107 \\
0.9906 & 175 & 0.6 & 56.1 & 57.4 & 2.3726 \\
0.9906 & 175 & 0.5 & 90 & 88.3 & 1.8532 \\
1.0502 & 215 & 0.4 & 82.3 & 81.1 & 1.4007 \\
1.0595 & 225 & 0.3 & 29.4 & 30.6 & 3.9273 \\
1.1025 & 240 & 0.2 & 45.6 & 44.8 & 1.7129 \\
1.1293 & 265 & 0.2 & 51.2 & 52.4 & 2.3615 \\
\hline
\end{tabular}

$\mathrm{V}=$ voltage at transformer bus before switching, L.L. = line length, $\Phi_{\mathrm{r}}=$ remanent flux, B.S. $A_{H I}=$ the best switching angle obtained by the harmonic index, B.S. $A_{\mathrm{ANN}}=$ the best switching angle obtained by the ANN, and Error = switching angle error.

\section{Conclusion}

This paper describes a new approach to determine the optimum switching instants to reduce harmonic overvoltages due to transformer energization during power system restoration. The proposed method is based on the harmonic index which integrates the key parameters of overvoltages generation. The minimum value of this index is corresponding to the best switching time for the transformer energization. On the other hand, for real time application, the paper presents a procedure for evaluating the best switching condition using an ANN based on LevenbergMarquardt second order training method. It has been adopted for obtaining small mean square error without losing generalization capability of ANN. Training ANN is based on equivalent circuit parameters to achieve good generalization capability for trained ANN. Simulation results confirm the effectiveness and accuracy of the proposed harmonic index and ANN scheme.

\section{References}

1. Adibi, M.M. and Kafka, R.J., Power System Restoration Issues, IEEE Computer Application in power, 4: 19-24, 1991.

2. Adibi, M.M., Alexander, R.W., and Avramovic, B., "Overvoltage control during restoration," IEEE Trans. Power Syst., 7: 14641470, 1992.

3. Ketabi, A., Ranjbar, A.M., and Feuillet, R., "Analysis and Control of Temporary Overvoltages for Automated Restoration Planning," IEEE Trans. Power Delivery, 17: 1121-1127, 2002.

4. Morin, G., Service restoration following a major failure on the hydroquebec power system, IEEE Trans. Power Delivery, 2: 454463, 1987.

5. Bourgault, O., and Morin, G., Analysis of harmonic overvoltage due to transformer saturation following load shedding on hydroquebec-NYPA $765 \mathrm{kV}$ interconnection, IEEE Trans. Power Delivery, 5: 397-405, 1990.

6. Sadeghkhani, I., Using Artificial Neural Network for Estimation of Switching and Resonance Overvoltages during Bulk Power System Restoration, M.Sc. dissertation, Department of Electrical Engineering, University of Kashan, 2009.
7. Sybille, G., Gavrilovic, M.M., Belanger, J., and Do, V.Q., Transformer saturation effects on EHV system overvoltages, IEEE Trans. Power App. Syst., PAS-104: 671-680, 1985.

8. Brunke, J.H., and Fröhlich, K.J., Elimination of Transformer Inrush Currents by Controlled Switching-Part I: Theoretical Considerations, IEEE Trans. on Power Delivery, 16: 276 - 280, 2001.

9. Dantas, K., Fernandes, D., Neves, L.A., Souza, B.A., and Fonseca, L., Mitigation of switching overvoltages in transmission lines via controlled switching, IEEE Power and Energy Society General Meeting-Conversion and Delivery of Electrical Energy in the 21st Century, pp. 1-8, 2008.

10. Thukaram, D., Khincha, H.P., Khandelwal, S., "Estimation of switching transient peak overvoltages during transmission line energization using artificial neural network," Electric Power System Research, 76: 259-269, 2006

11.Hagan, M.T., Menhaj, M.B., "Training feedforward networks with the Marquardt algorithm," IEEE Trans. Neural Network, 5: 989993, 1994. 
12. Hayashi, T., et al., Modeling and simulation of black start and restoration of an electric power system. Results of a questionnaire, Electra, 131: 157-169, 1990

13. Fink, L.H., Liou, K.L., and Liu, C.C., From generic restoration actions to specific restoration strategies, IEEE Trans. Power Syst., 10: 745-752, 1995 .

14. Sybille, G., Brunelle, P., Hoang, L., Dessaint, L.A., and Al-Haddad, K., "Theory and applications of power system blockset, a MATLAB/Simulink-based simulation tool for power systems," in Proc. IEEE Power Eng. Soc. Winter Meeting, 774-779, 2000.

15.Duro, M.M., "Damping Modelling in Transformer Energization Studies for System Restoration: Some Standard Models Compared to Field Measurements," in Proc. IEEE Bucharest Power Tech Conference, Bucharest, Romania, 2009.

16. Boliaris, P.G., Prousalidis, J.M., Hatziargyriou, N.D., and Papadias, B.C., "Simulation of long transmission lines energization for black start studies," in Proc. 7th Mediterranean Electrotechn. Conf., 1093-1096, 1994.

17. Haykin, S., Neural Network: A Comprehensive Foundation, 2nd ed., Prentice Hall, 1998.

18. Wunderlich, S., Adibi, M.M., Fischl, R., and Nwankpa, C.O.D., "An approach to standing phase angle reduction," IEEE Trans. Power Syst., 9: 470-478, 1994. 